\title{
Article
}

\section{Updating Standards of Facial Growth in Romanian Children and Adolescents Using the Anthropometric Method-A Pilot Study}

\author{
Emilia Ogodescu ${ }^{1, *}$, Malina Popa ${ }^{1, *}$, Magda Luca ${ }^{1}$, Andreea Igna ${ }^{1}$, Mariana Miron ${ }^{2}$ (D), Krisztina Martha ${ }^{3, *}$, \\ Anca Tudor ${ }^{4}$ and Carmen Todea ${ }^{2}$
}

Citation: Ogodescu, E.; Popa, M.; Luca, M.; Igna, A.; Miron, M.; Martha, K.; Tudor, A.; Todea, C. Updating Standards of Facial Growth in Romanian Children and Adolescents Using the Anthropometric Method-A Pilot Study. Int. J. Environ. Res. Public Health 2021, 18, 5288. https://doi.org/10.3390/ ijerph18105288

Academic Editors: Alessandro Nota and Simona Tecco

Received: 16 March 2021

Accepted: 12 May 2021

Published: 16 May 2021

Publisher's Note: MDPI stays neutral with regard to jurisdictional claims in published maps and institutional affiliations.

Copyright: (c) 2021 by the authors. Licensee MDPI, Basel, Switzerland. This article is an open access article distributed under the terms and conditions of the Creative Commons Attribution (CC BY) license (https:// creativecommons.org/licenses/by/ $4.0 /)$
1 Pediatric Dentistry Research Center, Department of Pediatric Dentistry, Faculty of Dentistry, "Victor Babes" University of Medicine and Pharmacy, Eftimie Murgu Square No. 2, 300041 Timisoara, Romania; luca.magda@umft.ro (M.L.); igna.andreea@umft.ro (A.I.)

2 Department of Oral Rehabilitation and Dental Emergencies, Faculty of Dentistry, "Victor Babes" University of Medicine and Pharmacy, Eftimie Murgu Square No. 2, 300041 Timisoara, Romania; miron.mariana@umft.ro (M.M.); todea.darinca@umft.ro (C.T.)

3 Department of Orthodontics, Faculty of Dentistry, "George Emil Palade” University of Medicine, Pharmacy, Science, and Technology of Târgu Mureș, Gheorghe Marinescu Street No. 38, 540142 Târgu Mureș, Romania

4 Department of Functional Sciences, Faculty of Medicine, “Victor Babes" University of Medicine and Pharmacy, Eftimie Murgu Square No. 2, 300041 Timisoara, Romania; atudor@umft.ro

* Correspondence: ogodescu.emilia@umft.ro (E.O.); popa.malina@umft.ro (M.P.); krisztina.martha@umfst.ro (K.M.); Tel.: +40-723-330-890 (E.O.)

Abstract: The anthropometric method is an important tri-dimensional and non-invasive assessment instrument for accurate diagnosis in paedodontics, orthodontics, and other medical fields. Our aim was to propose a technique that is accessible for clinicians and to determine the reference values for Romanian children and adolescents for the facial parameters selected. We proposed three basic instruments: a plastic compass, a ruler, and a digital caliper. Eighty-five children and adolescents (62\% girls and 38\% boys), aged between 3.5 and 14.5 years, were included in the study. We selected eight transversal, 12 vertical, and three sagittal measurements. Facial indices, according to Farkas L.G., were directly determined. The correlations between facial and general growth parameters, using Pearson correlation coefficient, for the entire sample were significant, direct, and strong for the following: Zy-Zy, Go-Go, N-Gn, N-Sn, Sto-Gn, Tr-Gn, Tr-Sn, Tr-Tr (r = 0.526-0.925, $p<0.001$ ), and insignificant for Sn-Sto $(r=0.099-0.124, p>0.354)$. The highest correlation coefficient is exhibited by TrGn ( $r=0.893$ with height and $r=0.925$ with weight). When performing a gender related comparison, we noticed that the vertical and transversal linear parameters and some facial indices are increased in boys $(p<0.05)$, depending on the age group. The simplified anthropometric technique represents an accessible method to every clinician, bringing important information related to dentofacial growth, diagnosis, and treatment planning in dentistry.

Keywords: facial growth; non-invasive measurements; anthropometry; reference values; healthy children and adolescents; orthodontic diagnosis; interceptive orthodontics

\section{Introduction}

Because dentists and, in particular, orthodontists, are involved in the development of not only the dentition, but the entire dentofacial complex, a practitioner may be able to manipulate the facial growth for the benefit of the patient. This fact requires a thorough understanding of both the pattern of normal growth and the mechanisms that underlie it [1]. In order to achieve the above-mentioned goal, dentists must have sufficient knowledge regarding the normal growth of the dentofacial complex and proper instruments represented by up-to-date standards.

Every person's face is a custom-made original [2]. Enlow D.H. (1996) explained the biologic rationale underlying common variations in facial features in Essentials of Facial 
Growth, taking three general features into account: (1) different facial types as they relate to variations in the development of overall form and shape of the whole head, (2) gender related developmental facial differences, and (3) child and adult facial differences [2].

In 1930, Scammon described four growth curves for distinct tissues of the human body: the genital, the general, the neural, and the lymphoid growth curve [1,3]. Facial growth, including skeletal and soft-tissue growth, follows the general somatic growth; it respects the cranio-caudal growth principle [1,3]. Graber T.M. determined the quantity of the craniofacial growth for different age groups, expressed in percentages. In the 1-5-year group, the cranium increases by $85 \%$, the maxilla by $45 \%$, and the mandible by $40 \%$; in the 5-10-year group, the cranium only modifies with $11 \%$, the maxilla with $20 \%$ and the mandible with $25 \%$. In the larger age interval ranging from 10 to 20 years, the cranium expends by only $4 \%$, while the maxilla and mandible increase by $35 \%$ each $[3,4]$.

In this context, it is important to know which are the values that practitioners should use as reference values and what is their meaning [5]. Our research started from the questions of how these standards were developed, and which are the values that practitioners use as standards. We tried to contribute to the development of reference values for Romanian growing population.

Craniofacial anthropometry is an objective technique, which is based on a series of linear and angular measurements and proportions; it facilitates the characterization of phenotypic variation and the evaluation of dysmorphology and dimensional variation during growth $[6,7]$. The assessment of craniofacial deformities by only visual examination is termed anthroposcopy; the method is highly subjective, and it does not provide quantitative data to the examiner $[6,8]$. The limits of anthropometry are represented by the sensitivity of some tissues to direct measurements, compressibility of soft tissues, differences of thicknesses and consistency of different tissues, and pressure of the instrument during measurement [9]. However, the method also has numerous advantages; it is a tri-dimensional technique that can deliver longitudinal data that are important for the orthodontic diagnosis and the craniofacial growth studies.

These direct facial measurements are effective and inexpensive, but difficult to perform, time consuming, and not conducive to a busy clinical practice. Recent advancements in technological field made the implementation of tri-dimensional photography or stereophotogrammetry possible, and a solution for the main disadvantages [10]. However, there is a lack of data for different age groups, until the new techniques can be widely used.

A recognized name in the field of craniofacial anthropometrics, Farkas L.G., who studied Canadian and Nordic European populations, mentioned that the craniofacial growth is predictable, however reference values should not be used for more than 20 years [5,11].

The craniofacial anthropometry can be used in many areas, such as the management of patients with cleft lip and palate (still lacking the reference values in infants), anaplasthology (possible to combine anthropometry and computer-aided design and manufacture technology for fabrication of epitheses), forensics (comparing recordings from video surveillance systems with manual measurements of the suspect subject), genetics and paediatrics (Down syndrome, plagiocephaly, craniosynostosis, and growth hormone deficit) for an early diagnosis of the anomalies and ergonomic product design $[6,10,12-16]$. Some authors determined an ameliorated facial profile due to improved mandibular development after treatment with growth hormone [17].

Proffit W. presented the commonly used measurement instruments (bow calipers and straight calipers) and pointed out the most important data of Farkas L.G. that were acquired by anthropometric measurements (linear and angular measurements, and facial indices) in young adults [1]. Clinicians need updated reference values for different populations, as determined on larger samples [18]. One study published in Canada (2007), compared the values between Americans of Caucasian origin and the Americans with African origin; significant differences in nasal and orbital region were recorded, which confirmed the necessity of reference values for each particular race [19]. 
The pattern and growth rate, the peripubertal growth spurt, and the differences between boys and girls were determined in a mixed longitudinal study, on individuals aged from six to 17 years, in Columbian mestizos; regional differences were recorded [20]. Anthropometric measurements for studies on facial growth have been also performed in Romania some decades ago by Firu P., Boboc G., Milicescu V., and Milicescu I.D. They used the measurements that were introduced by Rainer in 1937 and instruments developed by Martin and Firu [21-23].

Today, several competing methods are available for capturing and quantifying facial surface morphology, including two-dimensional photogrammetry. The recent development of facial tri-dimensional data acquisition (the accuracy and reliability using them being checked) reduced the time needed for assessment and increased the number of individuals of different age groups that can be included in studies [24-36]. Most of them were conducted on adults, highlighting the importance of our study on different age groups of Romanian children and adolescents using the traditional method.

The aims of our study were: (1) to implement simplified anthropometric instruments, accessible for every clinician and demonstrate the possibility of using this technique in growth studies; (2) to present the reference values obtained for selected facial parameters for each age group; (3) to compare the results between females and males and to other population; and, (4) to update the somatic growth general parameters at different ages and determine the possible correlation between them and the ones that characterize facial growth.

\section{Materials and Methods}

2.1. Instruments Used for the Simplified Orthodontic Technique in Orthodontics, Pedodontics and Growth Studies

Because of the fact that achieving a complete set of anthropometric instruments or the devices that are needed for the tri-dimensional data acquisition is not possible in every country or in every dental clinic, we propose three basic instruments: (1) a plastic compass that has unsharp edges, (2) a ruler, which is up to $30 \mathrm{~cm}$ by $\mathrm{mm}$ graded, and (3) a digital caliper that is used for the study model analyses, with a slightly unsharp edges (Figure 1). The first two instruments are used together and preferred for every child, even for the ones who are noticeably young (3-7 years) or express some uncooperativeness. The third instrument is used for more precise measurements of the smaller dimensions, but it is not indicated in younger children because of the risk of damaging tegument or eyes. Besides of these instruments, we added a digital body weight scale and a height scale (composed of one horizontal rod that moves in vertical plane.

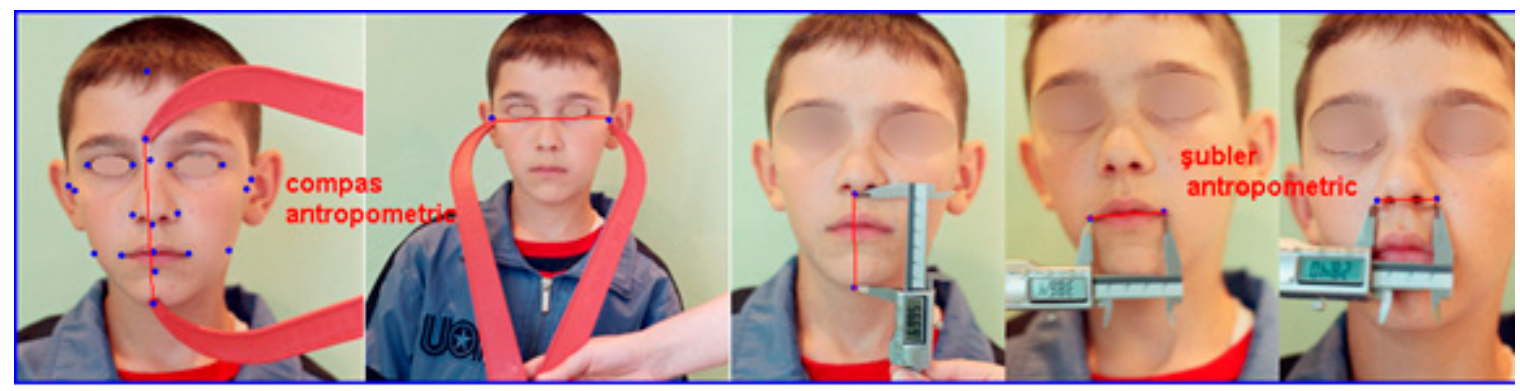

Figure 1. Specific instruments indicated for the simplified anthropometric method and examples of their use for the assessment of facial dimensions (plastic compass and digital caliper).

\subsection{Selection and Marking of the Main Reference Points}

We selected a number of eight transversal measurements, 12 vertical measurements, and three sagittal measurements, for which we presented standard values at different ages. We traced the cutaneous reference points (median, lateral and paramedian). The median reference points are: Trichion (Tri, Tr), Glabella (G, Gl), Nasion (N, Na), Subnasale (Sn), 
Labiale superior (Ls), Stomion (St, Sto), Labiale inferior (Li), Sublabiale (Sl) and Gnathion (Gn). The lateral reference points are: Zygion (Zy), Gonion (Go), Cheilion (Ch), Alar Point (Al), Entocanthion (En), Exocanthion (Ex), and Tragion (Tr, T). The point Cheilion (Ch) and the alar point $(\mathrm{Al})$ are considered to be paramedian by some authors [37] (Figure 2).
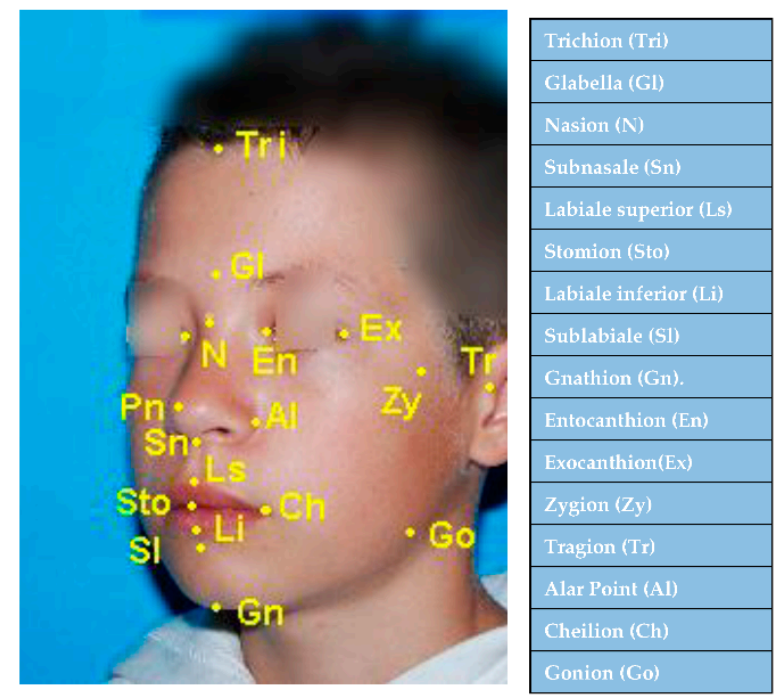

Figure 2. The median and lateral anthropometric points recommended for the anthropometric simplified technique.

\subsection{The Workiflow on Children of Different Ages}

The working flow includes two aspects: marking the reference points with a permanent marker or with an eyeliner and the measurement of the transversal, vertical, and sagittal parameters (Figures 3 and 4).

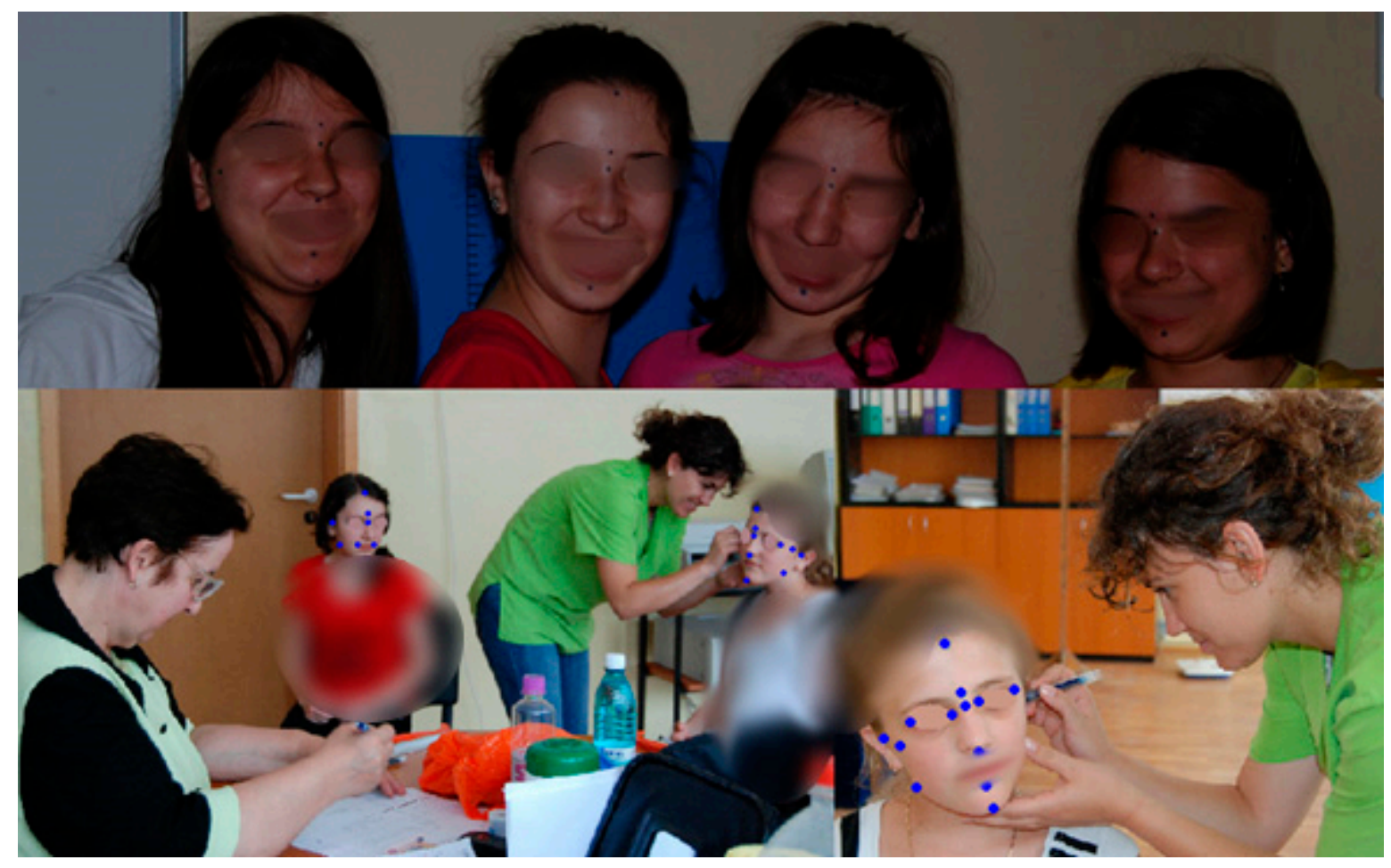

Figure 3. Marking of the median and lateral selected anthropometric reference points. 


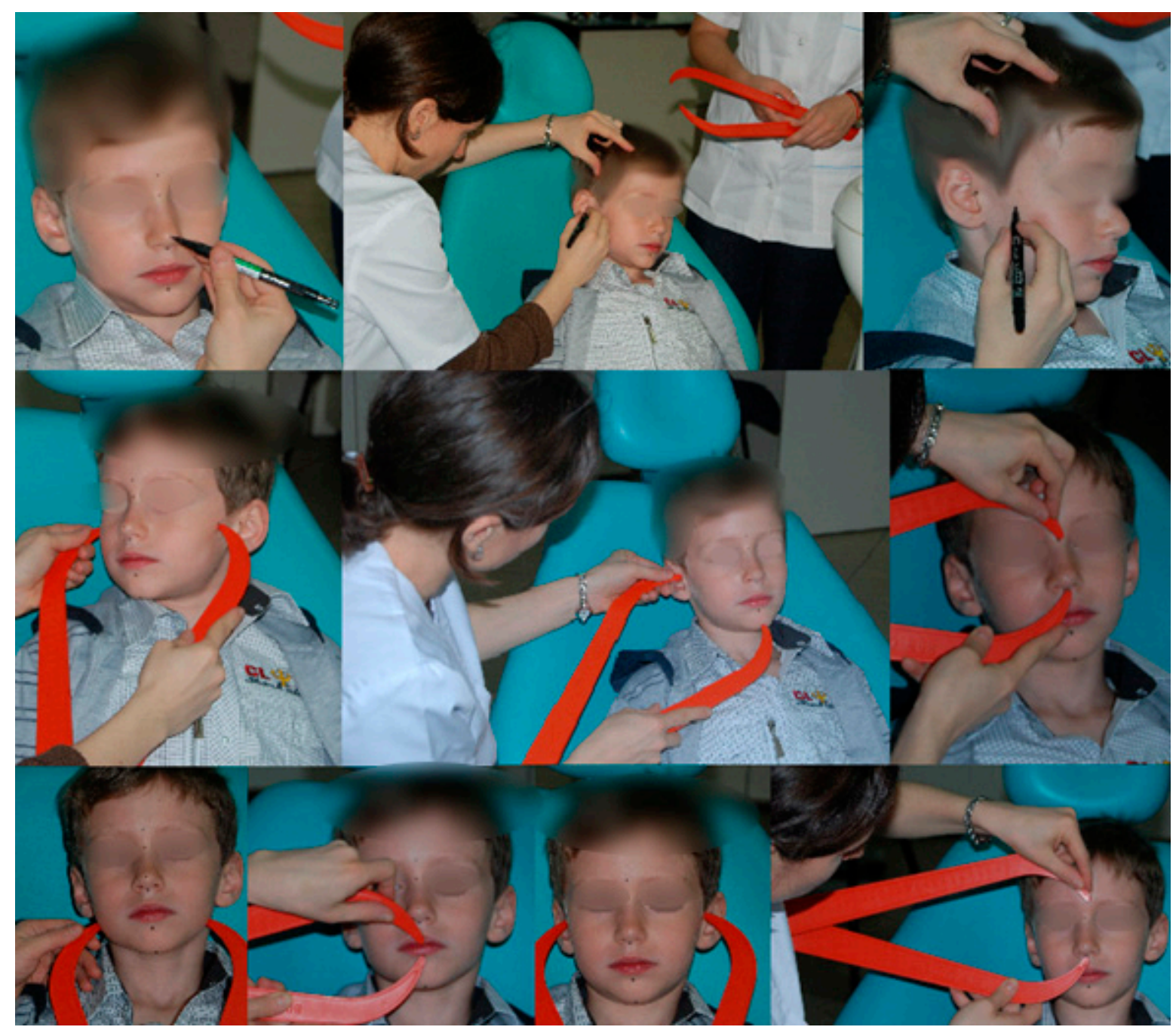

Figure 4. The simplified anthropometric technique used for the facial measurements.

One examiner and two assistants were involved in our study. One assistant has supervised the head position, with the Frankfurt horizontal plane being parallel to the floor, the correct location of landmarks, and the precision of measurements. The other assistant recorded the findings.

The selected transversal measurements are eight: Zygomatic width (maximal facial width; Zy-Zy); Gonial width (maximal mandibular width; Go-Go); Mouth width (maximal mouth width, measured from one mouth angle to the other; Ch-Ch), Nose width (maximal nose width; Al-Al); Nose basal width (En-En); the distance between the outer commissures of the eye fissures Ex-Ex); the distance between the $\mathrm{N}$ point and the middle of the left pupil; and, the width of the head between Tragus (Tr-Tr) [1,6,38].

The selected vertical measurements are 12, namely: Face height (N-Gn), upper face height (N-Sn), N-Sto, lower facial segment height (Sn-Gn), the height of both lower face segments, situated upper and lower to Sto (Sn-Sto and Sto-Gn), upper lip height (Sto-Ls) and lower lip height (Sto-Li), two segments of Sto-Gn, situated upper and lower to SI (Sto-Sl and Sl-Gn), G-Sn, and Tri-Sn.

The selected sagittal measurements are three: superior facial depth (Tr-N), middle facial depth (Tr-Sn), and lower facial depth (Tr-Gn).

The values that were obtained by measurements, as done in the three spatial planes, were used for determining the reference values for different dimensions and different ages. 


\subsection{Sample Description, Study Protocol and Data Analysis}

Our sample included 85 children and adolescents, from one nursery school and two schools, from Timisoara city and surroundings, Romania country. Fifty-three $(62 \%)$ were girls and 32 boys ( $38 \%$ ), aged between 3.5 and 14.5 years (Table 1$)$. The sample was best represented for the groups that were included in 11.5-14.5-year intervals.

Table 1. Age-groups distribution of the study subjects.

\begin{tabular}{ccc}
\hline Age-Group (Years) & Number of Subjects & Percentage (\%) \\
\hline $3.5-5$ & 14 & $16.5 \%$ \\
\hline $5-6.5$ & 11 & $13 \%$ \\
\hline $10-11.5$ & 11 & $13 \%$ \\
\hline $11.5-13$ & 28 & $33 \%$ \\
\hline $13-14.5$ & 21 & $24.5 \%$ \\
\hline
\end{tabular}

For the sample size calculation, we conducted a power analysis test with GPower3.1 application using the medium effect size, 0.05 level of significance, and $80 \%$ power.

The sample is representative, because it respects the proportions of age groups and categories according to BMI in the population (underweight $10.9 \%$, normal weight $72.7 \%$, overweight $10.9 \%$, and obese $5.5 \%$ ).

The time that is needed for each child measurement was $20 \mathrm{~min}$., with the exception of the children aged between 3.5 and 6.5 years; in the last cases, the time was increased according to the ability to cooperate with each child.

Participation in the study was voluntary. Written informed consent was obtained from all of the the participants' parents. The study has been carried out in accordance with the Declaration of Helsinki and it was approved by Institutional Ethics Committee (No 04/15.09.2008).

All of the measurements (expressed in $\mathrm{mm}$ ) were transferred to an Excel Database. The facial indices, which were selected from the international literature (used by Farkas L.G.), were directly determined, according to the proportions that are presented in the literature $[1,5,39]$.

The statistical analysis was performed using the SPSS version 17 . Descriptive statistics were calculated for subjects' characteristics (age, height, and weight) and facial parameters distributed in age groups (mean and standard deviation). We used the unpaired $t$ test for comparing the independent values between sexes and linear correlation analysis using the Pearson correlation coefficient in order to determine the correlation between the facial and the general growth parameters. A $p$ value of less than 0.05 was considered to be statistically significant.

The intra-examiner error has been calculated, by measuring a 25 children twice. The differences were non-significant, according to paired $t$-test $(p>0.05)$.

\section{Results}

Following the statistical analysis, we determined the mean, minimum, and maximum values for each measured facial parameter (Table 2).

The facial indices determined were: Facial Index (N-Gn/Zy-Zy); Mandible -facial width Index (Go-Go/Zy-Zy); Mandibular width-face height Index (Go-Go/N-Gn); Mandibular index or Jaw Index(Sto-Gn/Go-Go); Index of mouth width to jaw width (Ch-Ch/Go-Go); Index of upper lip height to mouth width (Sn-Sto/Ch-Ch); Index of lower lip height to mouth width (Sto-Sl/Ch-Ch); Upper Face Index (N-Sto/Zy-Zy); Lower face -face height Index (Sn-Gn/N-Gn); Mandible-face height Index (Sto-Gn/N-Gn); Mandible-upper face height Index (Sto-Gn/N-Sto); Mandible-lower face height Index(Sto-Gn/N-Sto); Index of upper to lower lip vermillion (Ls-Sto/Sto-Li); Index of upper to lower lip height (Sto-Sl/Sn- 
Sto); Index of upper lip to mandibular height (Sn-Sto/Sto-Gn); and, Index of Chin-face height (Sl-Gn/Sn-Gn) (Table 3).

Table 2. Facial dimensions in our study on different age groups.

\begin{tabular}{|c|c|c|c|c|c|}
\hline $\begin{array}{l}\text { Facial Di- } \\
\text { mensions } \\
(\mathrm{mm})\end{array}$ & $\begin{array}{l}\text { 3.5-5 Years } \\
\text { Mean } \pm \text { SD } \\
\text { (Min-Max) }\end{array}$ & $\begin{array}{l}\text { 5-6.5 Years } \\
\text { Mean } \pm \text { SD } \\
(\text { Min-Max) }\end{array}$ & $\begin{array}{l}\text { 10-11.5 Years } \\
\text { Mean } \pm \text { SD } \\
\text { (Min-Max) }\end{array}$ & $\begin{array}{c}\text { 11.5-13 Years } \\
\text { Mean } \pm \text { SD } \\
\text { (Min-Max) }\end{array}$ & $\begin{array}{l}\text { 13-14.5 Years } \\
\text { Mean } \pm \text { SD } \\
\text { (Min-Max) }\end{array}$ \\
\hline Zy-Zy & $114.79 \pm 6.15$ (105-126) & $116.91 \pm 6.02(109-128)$ & $126.18 \pm 3.87(122-135)$ & $126.25 \pm 6.52(112-138)$ & $129.43 \pm 5.48(120-140)$ \\
\hline Go-Go & $92.64 \pm 5.61(82-100)$ & $93.45 \pm 7.33(82-106)$ & $104.45 \pm 6.9(93-115)$ & $101.18 \pm 9.67(87-123)$ & $100.1 \pm 7.71(88-113)$ \\
\hline $\mathrm{Ch}-\mathrm{Ch}$ & $35.93 \pm 3.43(31-42)$ & $36.82 \pm 2.86(32-41)$ & $43.09 \pm 3.39(37-48)$ & $44.36 \pm 3.72(35-51)$ & $44.14 \pm 3.93(36-54)$ \\
\hline Al-Al & $27.43 \pm 1.6(26-32)^{\prime}$ & $27.27 \pm 1.56(26-31)$ & $30.36 \pm 2.01(26-33)$ & $29.64 \pm 2.77(25-37)$ & $30.29 \pm 2.08(26-33)$ \\
\hline En-En & - & - & $29.55 \pm 2.21(25-33)$ & $30.36 \pm 2.2(26-37)$ & $31.33 \pm 2.39(25-37)$ \\
\hline $\begin{array}{l}\text { N-middle } \\
\text { of Pupilla }\end{array}$ & - & & $29.91 \pm 0.83(29-31)$ & $29.86 \pm 2.74(24-35)$ & $31.9 \pm 1.87(28-35)$ \\
\hline Ex-Ex & $91.21 \pm 6.64(80-102)$ & $96.18 \pm 6.42(87-106)$ & $99.25 \pm 4.86(90-108)$ & $97.36 \pm 5.1(89-109)$ & $103.5 \pm 4.95(100-107)$ \\
\hline N-Gn & $86.29 \pm 2.37(83-91)$ & $87.55 \pm 4.32(82-96)$ & $100.09 \pm 3.48(95-105)$ & $102.25 \pm 6.22(91-114)$ & $105.19 \pm 5(92-111)$ \\
\hline N-Sn & $34.29 \pm 1.73(31-37)$ & $36.09 \pm 3.81(31-43)$ & $45.45 \pm 2.25(42-49)$ & $43.68 \pm 3.54(38-50)$ & $44.62 \pm 3.83(36-52)$ \\
\hline N-Sto & $52.86 \pm 2.57(49-57)$ & $55.36 \pm 3.07(51-60)$ & $63.82 \pm 3.09(58-68)$ & $62.96 \pm 4.35(54-71)$ & $64.43 \pm 3.11(60-71)$ \\
\hline Sn-Gn & $51.29 \pm 2.16(49-57)$ & $52.18 \pm 3.09(46-57)$ & $57.18 \pm 5.13(52-68)$ & $58.61 \pm 4.43(50-66)$ & $60 \pm 4.1(52-66)$ \\
\hline Sn-Sto & $17.43 \pm 1.87(14-20)$ & $19.55 \pm 4.34(12-29)$ & $19.09 \pm 3.21(14-25)$ & $18.68 \pm 2.83(12-23)$ & $18.9 \pm 1.41(16-21)$ \\
\hline Sto-Gn & $35.71 \pm 3.02(30-40)$ & $34.45 \pm 1.21(33-37)$ & $36.82 \pm 3.79(31-43)$ & $39.96 \pm 3.14(32-45)$ & $40.86 \pm 3.35(33-48)$ \\
\hline Sto-Ls & $6.25 \pm 1.55(3-9)$ & $6.41 \pm 1.71(4-10)$ & $7.55 \pm 1.04(6-9)$ & $6.89 \pm 1.49(4.5-11)$ & $6.67 \pm 1.56(4-10)$ \\
\hline Sto-Li & $7.89 \pm 1.39(5-10)$ & $6.45 \pm 1.13(5-9)$ & $8.91 \pm 1.76(6-11)$ & $9.07 \pm 1.22(6.5-12)$ & $8.9 \pm 1.79(7-13)$ \\
\hline Sto-Sl & $14.79 \pm 1.76(12-18)$ & $13.36 \pm 1.91(11-16)$ & $16.18 \pm 2.44(11-18)$ & $17.02 \pm 1.99(13-22)$ & $16.29 \pm 2.28(13-21)$ \\
\hline Sl-Gn & $20.93 \pm 3.41(13-25)$ & $21.09 \pm 2.77(17-26)$ & $20.64 \pm 2.46(18-25)$ & $23.55 \pm 4.07(16-35)$ & $24.57 \pm 3.16(17-30)$ \\
\hline G-Sn & $42.79 \pm 2.46(40-47)$ & $44.27 \pm 3.93(38-50)$ & $59.09 \pm 4.53(52-69)$ & $53.75 \pm 4.97(47-69)$ & $55 \pm 3.41(48-61)$ \\
\hline Tri-Sn & $95.71 \pm 4.1(91-103)$ & $97 \pm 6.07(89-107)$ & $105.45 \pm 4.61(101-114)$ & $107.86 \pm 8.98(90-122)$ & $113 \pm 8.83(97-128)$ \\
\hline Tr-Gn & $112.14 \pm 4.85(104-120)$ & $112.55 \pm 5.35(105-122)$ & $128.45 \pm 4.2(124-137)$ & $128.36 \pm 6.93(119-147)$ & $130 \pm 6.38(120-145)$ \\
\hline Tr-Sn & $103.57 \pm 2.24(99-107)$ & $103.45 \pm 6.02(94-111)$ & $115.36 \pm 3.32(111-122)$ & $115.57 \pm 5.33(108-131)$ & $118.14 \pm 6.24(105-127)$ \\
\hline $\operatorname{Tr}-\mathrm{N}$ & $100.86 \pm 2.68(94-104)$ & $101 \pm 3.44(95-105)$ & $110.55 \pm 3.53(106-119)$ & $112.54 \pm 5.1(102-125)$ & $114.38 \pm 4.75$ (107-121) \\
\hline $\operatorname{Tr}-\operatorname{Tr}$ & $121.21 \pm 4.37(112-126)$ & $122.36 \pm 5.1(115-131)$ & $130.64 \pm 4.84(121-137)$ & $132.25 \pm 6(120-144)$ & $134.38 \pm 6.36(122-145)$ \\
\hline
\end{tabular}

Table 3. Facial anthropometric indices in our study on different age groups.

\begin{tabular}{|c|c|c|c|c|c|}
\hline $\begin{array}{c}\text { Facial } \\
\text { Anthropometric } \\
\text { Indices }\end{array}$ & $\begin{array}{l}\text { 3.5-5 Years } \\
\text { Mean } \pm \text { SD } \\
(\text { Min-Max) }\end{array}$ & $\begin{array}{l}5-6.5 \text { Years } \\
\text { Mean } \pm \text { SD } \\
(\text { Min-Max) }\end{array}$ & $\begin{array}{l}\text { 10-11.5 Years } \\
\text { Mean } \pm \text { SD } \\
\text { (Min-Max) }\end{array}$ & $\begin{array}{l}\text { 11.5-13 Years } \\
\text { Mean } \pm \text { SD } \\
\text { (Min-Max) }\end{array}$ & $\begin{array}{l}\text { 13-14.5 years } \\
\text { Mean } \pm \text { SD } \\
\text { (Min-Max) }\end{array}$ \\
\hline N-Gn/Zy-Zy & $\begin{array}{c}75.34 \pm 3.87 \\
(70-80.95)\end{array}$ & $\begin{array}{c}75 \pm 4.25 \\
(67.97-81.82)\end{array}$ & $\begin{array}{c}79.38 \pm 3.49 \\
(73.33-84.55)\end{array}$ & $\begin{array}{c}81.07 \pm 4.46 \\
(70.45-93.33)\end{array}$ & $\begin{array}{c}81.33 \pm 3.55 \\
(75.41-88.33)\end{array}$ \\
\hline Go-Go/Zy-Zy & $\begin{array}{r}80.84 \pm 5.32 \\
(69.84-91.67)\end{array}$ & $\begin{array}{c}79.88 \pm 3.52 \\
(75.23-85.45)\end{array}$ & $\begin{array}{c}82.77 \pm 4.59 \\
(75-88.8)\end{array}$ & $\begin{array}{c}80.03 \pm 4.71 \\
(72-89.15)\end{array}$ & $\begin{array}{l}77.26 \pm 3.43 \\
(70.31-82.31)\end{array}$ \\
\hline Go-Go/N-Gn & $\begin{array}{c}107.48 \pm 7.72 \\
(94.25-117.65)\end{array}$ & $\begin{array}{c}106.83 \pm 7.81 \\
(92.13-121.84)\end{array}$ & $\begin{array}{c}104.51 \pm 8.27 \\
(91.35-116.16)\end{array}$ & $\begin{array}{c}99.07 \pm 8.84 \\
(83.93-119.79)\end{array}$ & $\begin{array}{c}95.24 \pm 6.95 \\
(81.08-105.71)\end{array}$ \\
\hline Sto-Gn/Go-Go & $\begin{array}{c}38.7 \pm 4.21 \\
(31.96-46.34)\end{array}$ & $\begin{array}{c}37.1 \pm 3.54 \\
(31.13-43.9)\end{array}$ & $\begin{array}{l}35.36 \pm 4.19 \\
(30.1-45.26)\end{array}$ & $\begin{array}{c}39.83 \pm 4.66 \\
(28.57-47.13)\end{array}$ & $\begin{array}{c}41.01 \pm 4.34 \\
(34.02-51.14)\end{array}$ \\
\hline $\mathrm{Ch}-\mathrm{Ch} / \mathrm{Go}-\mathrm{Go}$ & $\begin{array}{l}38.77 \pm 2.66 \\
(33.7-42.42)\end{array}$ & $\begin{array}{c}39.47 \pm 2.61 \\
(36.79-44.09)\end{array}$ & $\begin{array}{c}41.33 \pm 3.23 \\
(35.92-46.94)\end{array}$ & $\begin{array}{c}44.04 \pm 3.87 \\
(36.52-52.22)\end{array}$ & $\begin{array}{c}44.19 \pm 3.48 \\
(38.53-53.47)\end{array}$ \\
\hline Sn-Sto/Ch-Ch & $\begin{array}{c}48.77 \pm 5.79 \\
(39.02-60.61)\end{array}$ & $\begin{array}{c}52.78 \pm 9.1 \\
(37.5-70.73)\end{array}$ & $\begin{array}{l}44.88 \pm 10.2 \\
(29.17-67.57)\end{array}$ & $\begin{array}{l}42.23 \pm 6.15 \\
(26.67-51.11)\end{array}$ & $\begin{array}{c}43.11 \pm 4.7 \\
(33.33-55.56)\end{array}$ \\
\hline Sto-Sl/Ch-Ch & $\begin{array}{c}41.4 \pm 5.71 \\
(32.43-54.84)\end{array}$ & $\begin{array}{c}36.27 \pm 4.05 \\
(29.73-41.03)\end{array}$ & $\begin{array}{c}37.74 \pm 6.4 \\
(25-46.15)\end{array}$ & $\begin{array}{l}37.19 \pm 8.95 \\
(0-52.38)\end{array}$ & $\begin{array}{l}37.17 \pm 6.45 \\
(27.66-58.33)\end{array}$ \\
\hline N-Sto/Zy-Zy & $\begin{array}{c}46.13 \pm 2.58 \\
(42.86-51.85)\end{array}$ & $\begin{array}{c}47.44 \pm 3.2 \\
(42.97-52.29)\end{array}$ & $\begin{array}{c}50.62 \pm 2.87 \\
(45.19-53.66)\end{array}$ & $\begin{array}{c}49.93 \pm 3.3 \\
(42.64-55.47)\end{array}$ & $\begin{array}{c}49.83 \pm 2.51 \\
(45.26-55.47)\end{array}$ \\
\hline $\mathrm{Sn}-\mathrm{Gn} / \mathrm{N}-\mathrm{Gn}$ & $\begin{array}{c}59.44 \pm 1.87 \\
(56.98-62.64)\end{array}$ & $\begin{array}{l}59.63 \pm 2.72 \\
(56.1-63.22)\end{array}$ & $\begin{array}{c}57.08 \pm 3.96 \\
(53-66.02)\end{array}$ & $\begin{array}{l}57.34 \pm 3.18 \\
(52.78-65.66)\end{array}$ & $\begin{array}{l}57.04 \pm 2.79 \\
(51.46-61.9)\end{array}$ \\
\hline Sto-Gn/N-Gn & $\begin{array}{c}41.38 \pm 3.22 \\
(34.88-46.51)\end{array}$ & $\begin{array}{c}39.44 \pm 2.22 \\
(35.42-42.53)\end{array}$ & $\begin{array}{c}36.77 \pm 3.33 \\
(31-41.35)\end{array}$ & $\begin{array}{l}39.14 \pm 2.95 \\
(32.32-44.9)\end{array}$ & $\begin{array}{c}38.86 \pm 2.91 \\
(33.02-44.44)\end{array}$ \\
\hline Sto-Gn/N-Sto & $\begin{array}{l}67.68 \pm 6.15 \\
(56.6-77.55)\end{array}$ & $\begin{array}{c}62.41 \pm 4.11 \\
(56.67-68.63)\end{array}$ & $\begin{array}{c}57.73 \pm 5.54 \\
(48.44-65.15)\end{array}$ & $\begin{array}{c}63.61 \pm 4.91 \\
(52.24-74.58)\end{array}$ & $\begin{array}{c}63.58 \pm 6.37 \\
(51.47-78.69)\end{array}$ \\
\hline Sto-Gn/Sn-Gn & $\begin{array}{c}69.68 \pm 5.79 \\
(59.62-81.63)\end{array}$ & $\begin{array}{c}66.24 \pm 4.52 \\
(59.65-72)\end{array}$ & $\begin{array}{c}64.49 \pm 5.12 \\
(58.49-73.08)\end{array}$ & $\begin{array}{c}68.33 \pm 4.78 \\
(59.26-78.57)\end{array}$ & $\begin{array}{c}68.23 \pm 5.56 \\
(61.11-84.91)\end{array}$ \\
\hline Ls-Sto/Sto-Li & $\begin{array}{c}79.99 \pm 19.42 \\
(42.86-120)\end{array}$ & $\begin{array}{c}100.88 \pm 27.62 \\
(66.67-150)\end{array}$ & $\begin{array}{c}86.41 \pm 12.94 \\
(70-114.29)\end{array}$ & $\begin{array}{l}76.19 \pm 14.09 \\
(55.56-122.22)\end{array}$ & $\begin{array}{c}75.52 \pm 14.32 \\
(50-100)\end{array}$ \\
\hline
\end{tabular}


Table 3. Cont.

\begin{tabular}{cccccc}
\hline $\begin{array}{c}\text { Facial } \\
\text { Anthropometric } \\
\text { Indices }\end{array}$ & $\begin{array}{c}\text { 3.5-5 Years } \\
\text { Mean } \pm \text { SD } \\
\text { (Min-Max) }\end{array}$ & $\begin{array}{c}\text { 5-6.5 Years } \\
\text { Mean } \pm \text { SD } \\
\text { (Min-Max) }\end{array}$ & $\begin{array}{c}\text { 10-11.5 Years } \\
\text { Mean } \pm \text { SD } \\
\text { (Min-Max) }\end{array}$ & $\begin{array}{c}\text { 11.5-13 Years } \\
\text { Mean } \pm \text { SD } \\
\text { (Min-Max) }\end{array}$ & $\begin{array}{c}\text { 13-14.5 years } \\
\text { Mean } \pm \text { SD } \\
\text { (Min-Max) }\end{array}$ \\
\hline \multirow{2}{*}{ Sto-Sl/Sn-Sto } & $85.5 \pm 12$ & $70.76 \pm 15.55$ & $86.67 \pm 19.08$ & $89.87 \pm 22.92$ & $86.68 \pm 14.51$ \\
& $(70-106.25)$ & $(50-100)$ & $(61.11-121.43)$ & $(0-133.33)$ & $(66.67-125)$ \\
\hline \multirow{2}{*}{ Sn-Sto/Sto-Gn } & $49.08 \pm 6.39$ & $56.85 \pm 13.1$ & $52.19 \pm 9.23$ & $46.93 \pm 7.5$ & $46.55 \pm 4.79$ \\
& $(38.89-64.52)$ & $(34.29-85.29)$ & $(36.84-69.44)$ & $(27.27-60)$ & $(33.33-54.55)$ \\
\hline \multirow{2}{*}{ Sl-Gn/Sn-Gn } & $40.76 \pm 6.24$ & $40.58 \pm 6.04$ & $36.13 \pm 3.31$ & $40.28 \pm 7.07$ & $40.93 \pm 4.4$ \\
& $(26.53-48.98)$ & $(30.91-50)$ & $(32.14-41.67)$ & $(29.63-61.4)$ & $(31.48-50.85)$ \\
\hline
\end{tabular}

SD: standard deviation

In order to have a better image regarding variation with age of different parameters, we chose two commonly measured transversal parameters (facial width and mandibular width), one vertical (facial height), one sagittal (Tr-Gn), and two facial indices (Facial Index and Mandibular width-Face Height Index) to be represented in the form of Boxplot (Figures 5-10).

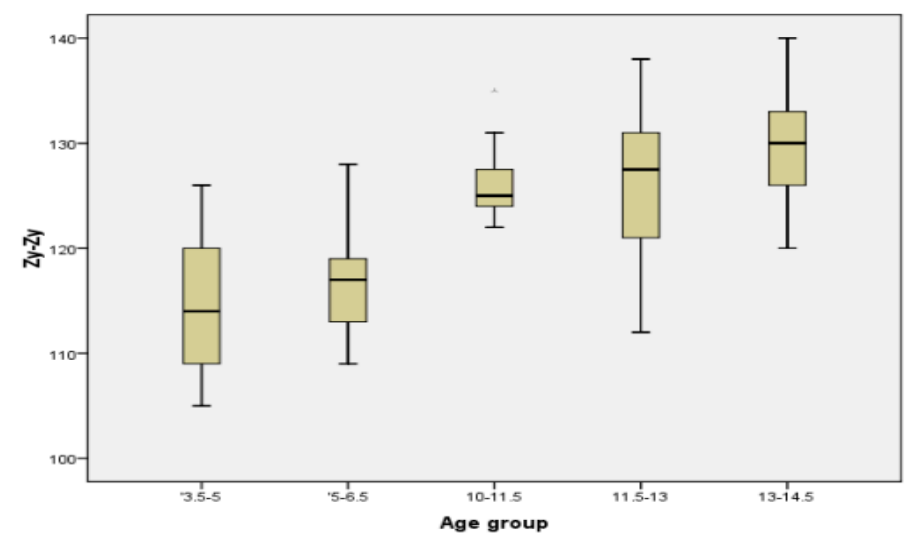

Figure 5. Boxplot for Zy-Zy values.

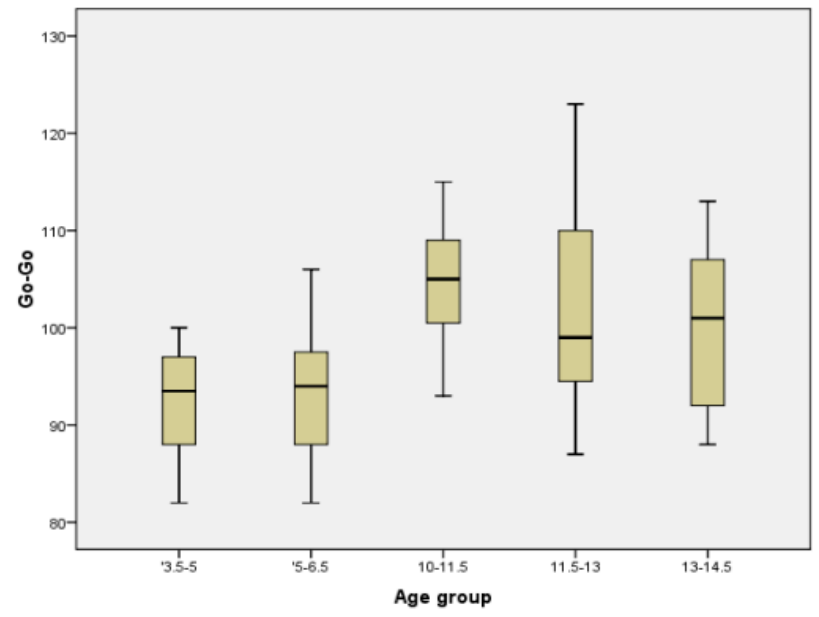

Figure 6. Boxplot for Go-Go values. 


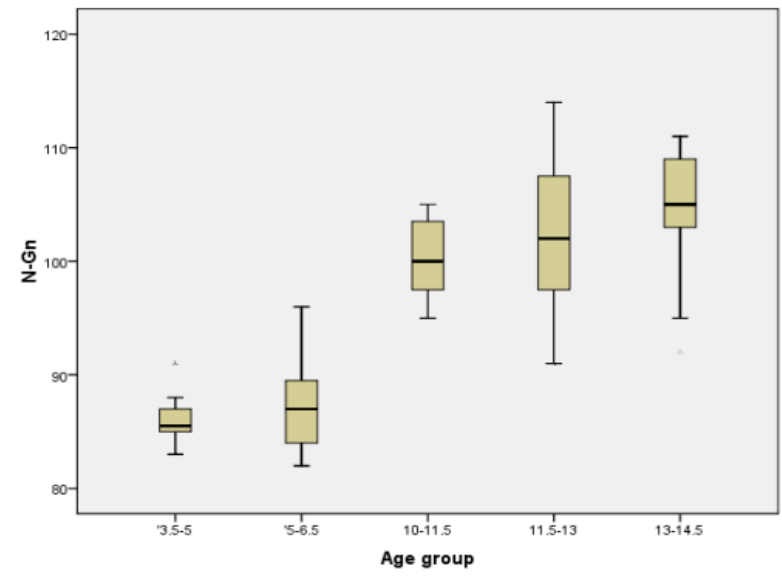

Figure 7. Boxplot for N-Gn values.

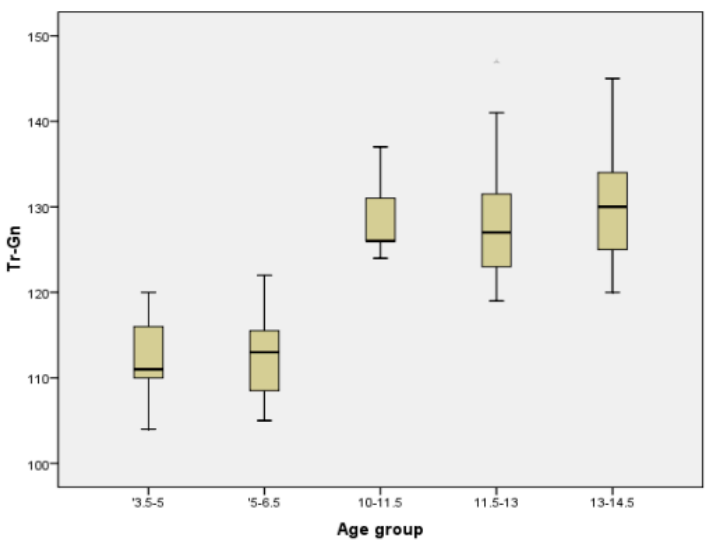

Figure 8. Boxplot for Tr-Gn values.

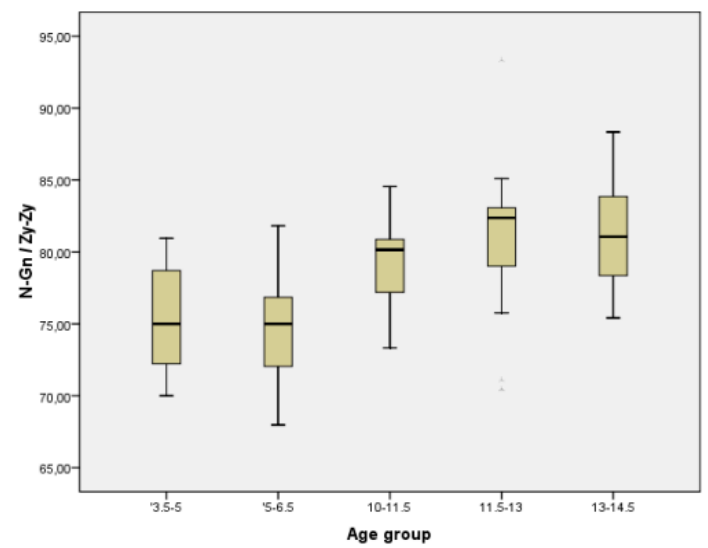

Figure 9. Boxplot for N-Gn/Zy-Zy values. 


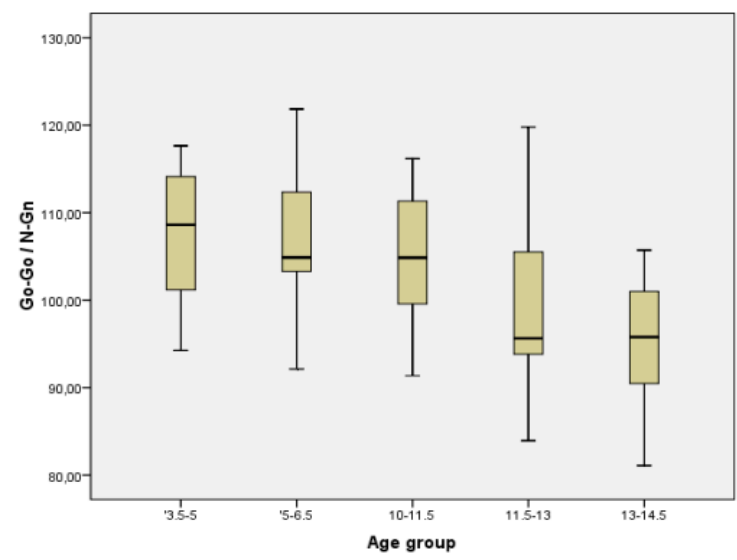

Figure 10. Boxplot for Go-Go/N-Gn values.

We determined the mean weight and height values, for one-year age intervals on a larger sample, including all of the values determined on children that participated in our dentofacial growth studies: the permanent teeth eruption study on a sample of 382 children (189 girls and 193 boys) aged between 3.5 and 15.5 years; the fotoanthropometric study on a sample of 156 children ( $55 \%$ girls and $45 \%$ boys) also aged between 3.5 and 15.5 years, and the present sample (Table 4).

Table 4. Age group distribution of the general growth parameters in study subjects.

\begin{tabular}{ccccc}
\hline \multirow{2}{*}{$\begin{array}{c}\text { Age Group } \\
\text { (Years) }\end{array}$} & $\begin{array}{c}\text { Height }(\mathbf{c m}) \\
\text { (Mean } \pm \text { SD) }\end{array}$ & $\begin{array}{c}\text { Weight }(\mathbf{k g}) \\
\text { (Mean } \pm \text { SD) }\end{array}$ & $\begin{array}{c}\text { Height }(\mathbf{c m}) \\
\text { (Mean } \pm \text { SD) }\end{array}$ & $\begin{array}{c}\text { Weight (kg) } \\
\text { (Mean } \pm \text { SD) }\end{array}$ \\
\hline $3.5-4.4$ & $107 \pm 4.97$ & $18.21 \pm 2.18$ & $106.67 \pm 4.93$ & $17.67 \pm 2.52$ \\
\hline $4.5-5.4$ & $110 \pm 5.77$ & $20.42 \pm 3.77$ & $112.86 \pm 6.41$ & $21.54 \pm 4.07$ \\
\hline $5.5-6.4$ & $118.75 \pm 3.69$ & $22.64 \pm 2.55$ & $126 \pm 7.79$ & $24.71 \pm 4.93$ \\
\hline $6.5-7.4$ & $122.67 \pm 6.43$ & $22.75 \pm 1.71$ & $124.75 \pm 6.58$ & $25.41 \pm 5.34$ \\
\hline $7.5-8.4$ & $132.67 \pm 4.95$ & $33.21 \pm 8.52$ & $132.57 \pm 7.39$ & $33.57 \pm 9.84$ \\
\hline $8.5-9.4$ & $133.18 \pm 5.91$ & $33.58 \pm 7.17$ & $135.39 \pm 8.77$ & $31.29 \pm 5.52$ \\
\hline $9.5-10.4$ & $139.27 \pm 5.7$ & $33.24 \pm 5.94$ & $133.29 \pm 12.11$ & $33.65 \pm 6.52$ \\
\hline $10.5-11.4$ & $149.83 \pm 8.35$ & $43.91 \pm 12.92$ & $147.33 \pm 6.41$ & $39.92 \pm 7.86$ \\
\hline $11.5-12.4$ & $154.56 \pm 8.25$ & $45.01 \pm 9.74$ & $152.74 \pm 7.09$ & $46.85 \pm 10.29$ \\
\hline $12.5-13.4$ & $157.47 \pm 6.71$ & $45.32 \pm 7.28$ & $161.33 \pm 9.45$ & $53.17 \pm 12.49$ \\
\hline $13.5-14.4$ & $161.08 \pm 5.3$ & $49.78 \pm 8.06$ & $164.25 \pm 5.28$ & $58.6 \pm 9.72$ \\
\hline $14.5-15.4$ & $159.5 \pm 2.12$ & $53.75 \pm 9.55$ & $165.83 \pm 11.58$ & $58.43 \pm 21.93$ \\
\hline & SD: standard deviation. & &
\end{tabular}

We compared the values between boys and girls using the unpaired $t$ test and taking the whole sample in account and determined significantly increased values for boys, regarding the following dimensions and facial indices: N-Gn $\left(0.041^{\mathrm{s}}\right)$; N-Sn $\left(0.031^{\mathrm{s}}\right)$; GSn $\left(<0.001^{\mathrm{s}}\right)$; Tri-Sn $\left(0.030^{\mathrm{s}}\right) ; \mathrm{N}-\mathrm{Gn} / \mathrm{Zy}-\mathrm{Zy}\left(0.026^{\mathrm{s}}\right)$; Go-Go/Zy-Zy $\left(0.010^{\mathrm{s}}\right)$; Go-Go/NGn $\left(0.002^{\mathrm{s}}\right)$; Sn-Gn/N-Gn $\left(0.001^{\mathrm{s}}\right)$; and, Sto-Gn/N-Gn $\left(0.049^{\mathrm{s}}\right)$. Other differences found were insignificant.

We also used the unpaired $t$ test for the age group of 11.5-13 years (where the number of cases for boys and girls of our sample is consistent), for comparing the values between two sexes. The value for boys were significantly increased, for the following dimensions and facial indices: $\mathrm{Zy}-\mathrm{Zy}\left(0.046^{\mathrm{s}}\right)$, Go-Go $\left(0.007^{\mathrm{s}}\right)$, Ch-Ch $\left(0.045^{\mathrm{s}}\right), \mathrm{Al}-\mathrm{Al}\left(0.049^{\mathrm{s}}\right)$, Sn-Gn $\left(0.031^{\mathrm{s}}\right)$, Sn-Sto $\left(0.001^{\mathrm{s}}\right)$, and $\operatorname{Tr}-\operatorname{Tr}\left(0.004^{\mathrm{s}}\right)$. The other differences for the facial dimensions 
were insignificant. Four from all of the determined facial indices presented increased values for boys from this age group: Go-Go/N-Gn $\left(0.029^{\mathrm{s}}\right)$, Sn-Sto/Ch-Ch $\left(0.024^{\mathrm{s}}\right)$, Sn-Sto/Sto-Gn $\left(0.030^{\mathrm{s}}\right)$, and Go-Go/Zy-Zy $\left(0.010^{\mathrm{s}}\right)$.

The correlations between the facial parameters and values that were obtained for height and weight for the entire sample were significant, direct, and strong for the following dimensions: Zy-Zy, Go-Go, N-Gn, N-Sn, Sto-Gn, Tr-Gn, Tr-Sn, and sTr-Tr $(r=0.526-0.925$, $p<0.001)$. There was an insignificant correlation for Sn-Sto $(\mathrm{r}=0.099-0.124, p>0.354)$. The higher correlation coefficient is exhibited by sagittal parameter $\operatorname{Tr}-\mathrm{Gn}(\mathrm{r}=0.893$ with height and $\mathrm{r}=0.925$ with weight) (Table 5).

Table 5. The correlations between facial parameters and general growth parameters.

\begin{tabular}{ccccc}
\hline \multirow{2}{*}{$\begin{array}{c}\text { Facial } \\
\text { Parameters }\end{array}$} & $\begin{array}{c}\text { Correlation } \\
\text { Coefficient }\end{array}$ & $p$-Value & $\begin{array}{c}\text { Correlation } \\
\text { Coefficient }\end{array}$ & $p$-Value \\
\cline { 2 - 5 } Zy-Zy & 0.827 & $<0.001$ & 0.805 & $<0.001$ \\
\hline Go-Go & 0.526 & $<0.001$ & 0.626 & $<0.001$ \\
\hline N-Gn & 0.938 & $<0.001$ & 0.895 & $<0.001$ \\
\hline N-Sn & 0.847 & $<0.001$ & 0.751 & $<0.001$ \\
\hline Sn-Sto & 0.124 & 0.354 & 0.099 & 0.444 \\
\hline Sto-Gn & 0.783 & $<0.001$ & 0.785 & $<0.001$ \\
\hline Tr-Gn & 0.893 & $<0.001$ & 0.925 & $<0.001$ \\
\hline Tr-Sn & 0.861 & $<0.001$ & 0.848 & $<0.001$ \\
\hline Tr-Tr & 0.814 & $<0.001$ & 0.720 & $<0.001$ \\
\hline
\end{tabular}

\section{Discussion}

We conducted a cross-sectional study to characterize the facial growth by dividing the sample in five age intervals. We included two groups: the younger group (3.5-6.5 years) when considering the importance of early diagnosis for interception in this age interval and the older group (10-14.5 years) because of the importance of quantifying the growth at the age of puberty, when there is an increased somatic growth spurt and increased facial growth, and the initiation of dentofacial orthopaedic treatments according to the right assessment and diagnosis.

Calculating the body mass index (BMI) for each child, we obtained a distribution in the sample that is considered to be acceptable according to the variable percentage, regarding the distribution of BMI in Romanian children and adolescents.

Overweight and obesity vary between $17 \%$ and $30 \%$, and they affect boys more than girls, particularly school age children than teenagers [40].

Another study determined that $8.29 \%$ of children are obese, while $12.84 \%$ of them are overweight, with boys being more likely to be obese; the highest prevalence has been observed among the 6-10 years' age group, while teenagers have recorded the lowest prevalence [41].

In a study on children aged 6-19 years, across the last decade (2006-2015), which included Timisoara, the prevalence of underweight children was 5\%/4.5\%/8.5\% (WHO/IOTF/CDC), while the prevalence of overweight (including obese) children was $28.3 \% / 23 \% / 23.2 \%$ (WHO/IOTF/CDC). This prevalence remained relatively stable over the last decade. Male gender, prepubertal age, and urban environment were the most relevant risk factors associated with overweight status in Romanian children [42].

Regarding the correlations among facial parameters and height and weight, there was one parameter (Sn-Sto) for which the correlation was insignificant, which suggested that the upper lip height has great interindividual variability. The strongest correlation with 
general growth was determined for Tr-Gn, which suggested the highest correlation with vertical and anterior mandibular growth.

When we started to assess the values of facial parameters at different ages and to determine the changes during the dentofacial growth, we should have begun by determining the reference values for the adults in that population. The values of facial dimensions in adults were published for the first time in 1981 in "Anthropometry of head and face in medicine; they were recommended by Profitt as up-to-date for the Caucasian population, and they are still currently used [1].

Non-invasive methods, like photographic and anthropometric, are preferred for both healthy subjects and patients, especially at young ages. After determining the reference values for our sample, we compared a part of the results with data from national literature (from some decades ago, published in 2001 and 2009) and from the international literature $[1,21,23]$.

In Romania, Boboc G. determined the interzygomatic distance in a study on 100 adult crania. The recorded values were $131.1 \mathrm{~mm}( \pm 5.54 \mathrm{~mm})$ in men and $124.46 \mathrm{~mm}( \pm 4.35 \mathrm{~mm})$ in women. This means that, in adults, an important difference between sexes appears, when we take the facial width into account $(7 \mathrm{~mm}$ larger in males). The cutaneous measurement values are higher with 10-15 $\mathrm{mm}$ than the osseous ones; the interzygomatic distance increases by $20 \mathrm{~mm}$, starting from seven years to adulthood [23]. Proffit recommends smaller reference values for the interzygomatic distance, which makes direct facial measurements on young adults. This is $137 \mathrm{~mm}( \pm 4.3 \mathrm{~mm})$ in males and $130 \mathrm{~mm}( \pm 5.3 \mathrm{~mm})$ in females [1]

In our study, including the 3.5-14.5 years age interval, the mean interzygomatic distance (Zy-Zy) ranges between 115-129.5 mm. Boboc G. (in Romania) determined. in a sample (3-7 years age interval), a value raging between 110.3 and $118.93 \mathrm{~mm}$ for boys, and between 109 and $118.42 \mathrm{~mm}$ for girls. Milicescu (in Romania) determined (7-11 years age interval) a value between $125.5 \mathrm{~mm}$ and $129.6 \mathrm{~mm}$ for boys, and between $122.9 \mathrm{~mm}$ and $126.3 \mathrm{~mm}$ for girls $[1,21,23]$.

The gonial width (Go-Go) that was determined by Farkas L.G. on adult population was $97 \mathrm{~mm}( \pm 5.8 \mathrm{~mm}) \mathrm{mm}$ for males and $91 \mathrm{~mm}( \pm 5.9 \mathrm{~mm})$ for females. Boboc (in Romania) determined on subjects included in 3-7 years age interval, a value of $80-87 \mathrm{~mm}$ for boys and 78-85 mm for girls. Milicescu (in Romania) determined (7-11 years age interval) a value between $98.3-98.4 \mathrm{~mm}$ for boys and $93.6-94 \mathrm{~mm}$ for girls. In our study, the mean value for the same parameter ranged between $92.5 \mathrm{~mm}$ and $104.5 \mathrm{~mm}$, with an increased value when compared to those that were presented by Farkas, Milicescu, and Boboc $[1,21,23]$. Comparing our values with the values that were obtained by Milicescu, we noticed the decreased values between N-Gn (86-105 mm in our study, as compared to $101.5-108.5 \mathrm{~mm}$ for boys and $96.6-105.7 \mathrm{~mm}$ for girls), N-Sn (34-45.5 $\mathrm{mm}$ in our sample compared to $46.9-52.3 \mathrm{~mm}$ for boys and $42.4-50 \mathrm{~mm}$ for girls), and N-Sto (53-64.5 mm in our study when compared to $61.5-71.4 \mathrm{~mm}$ for boys and $65.6-68.9 \mathrm{~mm}$ for girls), as well as increased values for $\operatorname{Tr}-\operatorname{Tr}$ (121-134.5 $\mathrm{mm}$ in our study as compared to $116.8-117.4 \mathrm{~mm}$ for boys and 113.3-114.1 $\mathrm{mm}$ for girls) in our sample [1,21].

Other mean values ranges for the whole sample of our study were then compared to the mean values of adults, as determined by Farkas, in order to have an overview of the results of our study. The mean value range for $\mathrm{Ch}-\mathrm{Ch}$ in our study was $36-44 \mathrm{~mm}$, compared to $53 \mathrm{~mm}$ for men and $50 \mathrm{~mm}$ for woman, as determined by Farkas. The smallest differences were assessed for parameters $\mathrm{Al}-\mathrm{Al}$ (27.5-30.5 $\mathrm{mm}$ in our study, when compared to $35 \mathrm{~mm}$ for men and $31 \mathrm{~mm}$ for women), En-En (29.5-31 mm as compared to $33 \mathrm{~mm}$ for men and $32 \mathrm{~mm}$ for women) and N-middle of Pupilla (30-32 $\mathrm{mm}$ as compared to $33 \mathrm{~mm}$ for men and $31 \mathrm{~mm}$ for women). The determined lower face height (Sn-Gn) values in our study ranged between 51-60 mm, compared to those of Farkas $(72 \mathrm{~mm}$ for men and $66 \mathrm{~mm}$ for women). We also compared the Upper lip vermillion and the Lower lip vermillion to the international reference values for adults (the determined Sto-Ls was 6-7.5 mm, as compared to $8.9 \mathrm{~mm}$ for men and $8.4 \mathrm{~mm}$ for women; the determined Sto-Li was 6.5-9 mm, when compared to $10.4 \mathrm{~mm}$ for men and $9.7 \mathrm{~mm}$ for women) [1]. 
The majority of data published in the international literature are for young adults. We compared the data of our study with reference values, for different population, with two of them being studied on children on 6-17 years age interval (Colombian mestizos) and 12-18 years age interval (boys from Nord of India) and the other two on Malaysian and Indo-Mauritian young adults, thus suggesting the existence of racial differences. [20,43-45]. Bizygomatic width for our sample ranged between $115 \mathrm{~mm}$ and $129.5 \mathrm{~mm}$, whereas, for Indo-Mauritian young adults, it was $144 \mathrm{~mm}$ for men and $140 \mathrm{~mm}$ for women, $136.3 \mathrm{~mm}$ for Malaysian young adults, $120-136 \mathrm{~mm}$ for Colombian mestizos boys, and 118-131 mm for mestizos girls and only $99.45 \mathrm{~mm}$ for Nord Indian boys. The gonial width ranged in our sample between $92.5-104.5 \mathrm{~mm}$, similar to 6-17 years' girls of Colombian mestizos (93-104 mm), whereas boys presented 95-108 mm range values, $105 \mathrm{~mm}$, respectively, $99 \mathrm{~mm}$ for Indo-Mauritian boys and girls and 83.5 for Nord Indian boys. The last-mentioned population had the smallest value. The determined Face height (N-Gn) in our sample was 86-105 mm, as compared to $94-117 \mathrm{~mm}$ and $92-109 \mathrm{~mm}$ for Colombian mestizos, boys, respectively, girls, $115.8 \mathrm{~mm}$, respectively, 110 for Indo-Mauritian, men and women, $115.6 \mathrm{~mm}$ for Malaysian adults, and 102.4 for Indian boys. The determined upper face height (N-Sn) range was 34-45.5 mm, whereas it was $52 \mathrm{~mm}$ for Malaysian and IndoMauritian young adults and 47.8 for Indian boys. The determined Nose width (Al-Al, 29.5-31 mm) was compared to Malaysian (39.2 mm) and Indo-Mauritian (32.8 mm for men and $29.5 \mathrm{~mm}$ for women) young adult values. We only compared the different parameter values from our sample with Indo-Mauritian young adults' values because of a lack of results in other studies (36-44 mm as compared with 47.9 for mouth width; 29.5-31 mm compared with $33.2 \mathrm{~mm}$ for nose basal width; 51-60 mm when compared with $65.9 \mathrm{~mm}$ for lower face height; 6-7.5 mm compared with 9.4 for upper lip vermillion; and, 6.5-9 mm compared with $11.5 \mathrm{~mm}$ for lower lip vermillion). We could not compare the N-middle of Pupilla and Tr-Tr because of a lack of values for these parameters in these studies [20,43-45].

The facial indices were compared to the values that were determined by Farkas (1987) in adults and in 4/5-year children (2003). In our study, for the 3.5-5-year-group (as compared to 4/5-year sample of Farkas), we noticed the following: the Facial Index $(\mathrm{N}-\mathrm{Gn} / \mathrm{Zy}-\mathrm{Zy})$ is decreased $(75.34 \pm 3.87$ compared to $87.6 \pm 4.2 / 86.7 \pm 3.6$ for boys and $86.8 \pm 4.9 / 88.3 \pm 4.8$ for girls); the Mandibular Index (Sto-Gn/Go-Go) is decreased $(38.7 \pm 4.21$ compared to $47.9 \pm 4.9 / 48.5 \pm 3.2$ for boys and $48.3 \pm 4.7 / 48.3 \pm 3.8$ for girls); the index of mouth width to jaw width (Ch-Ch/Go-Go) is decreased (38.77 \pm 2.66 compared to $45 \pm 3.9 / 46.7 \pm 2.4$ for boys and $46.7 \pm 7.4 / 46.2 \pm 3.1$ for girls); the index of upper lip height to mouth width (Sn-Sto/Ch-Ch) is decreased (48.77 \pm 5.79 compared to $50.2 \pm 4.8 / 48.1 \pm 4.3$ for boys and $49.4 \pm 4.8 / 48.0 \pm 5$ for girls); the index of lower lip height to mouth width (Sto-Sl/Ch-Ch) is increased (41.4 \pm 5.71 when compared to $38.8 \pm 4.5 / 37.5 \pm 3.4$ for boys and $38.1 \pm 4.4$ /38.4 \pm 3.3 for girls); the index of upper to lower lip vermillion (Ls-Sto/Sto-Li) is decreased (79.99 \pm 19.42 compared to $110.6 \pm 25.2 / 103.9 \pm 17.3$ for boys and $113.0 \pm 23.7 / 107.3 \pm 21.9$ for girls).; the index of upper to lower lip height (Sto-Sl/Sn-Sto) is increased (85.5 \pm 12 compared to $77.7 \pm 6.1 / 78.3 \pm 5.9$ for boys and $77.7 \pm 8.8 / 80.5 \pm 7.3$ for girls); and, the index of upper lip to mandibular height (Sn-Sto/Sto-Gn) is increased (49.08 \pm 6.39 as compared to $47.7 \pm 5.4 / 46.3 \pm 3.9$ for boys and $46.6 \pm 4.3 / 45.8 \pm 3.3$ for boys). The mean values ranges for the whole sample of our study were then compared to the mean values of adults, as determined by Farkas, in order to have an overview of the results of our study (the determined Facial Index (N-Gn/Zy-Zy) range was 75.3-81.3, as compared to $88.5 \pm 5.1$ in adult men and $86.2 \pm 4.6$ in adult women, showing that this increases with age and the quantity of increase to adulthood; the Mandible-Face width (Go-Go/Zy-Zy) range was 77.2-82.7, compared to $70.8 \pm 3.8$ for adult men and $70.1 \pm 4.2$ for adult females, presenting a decrease with age; the Mandibular width-Face Height (Go-Go/N-Gn) range was 95.2-107.48, compared to $80.3 \pm 6.8$ for adult men and $81.7 \pm 6.0$ for adult women, demonstrating a higher decrease with age; the Upper face Index (N-Sto/Zy-Zy) range was 46.13-50.62, compared to $54 \pm 3.1$ for adult men and $52.4 \pm 3.1$ for adult women, showing an slight increase with 
age; determined Mandibular Index (Sto-Gn/Go-Go) range was 35.36-41.01 compared to $51.8 \pm 6.2$ for adult men and $49.8 \pm 4.8$ for adult women, suggesting a consistent increase with age; and, the Mandible-Face Height range was (Sto-Gn/N-Gn) range was 36.77-41.38, compared to $41.2 \pm 2.3$ for adult male and $40.4 \pm 2.1$ for adult female, which suggests that this proportion only suffers a slight increase to adulthood). [1,5].

\subsection{Study Limitations}

We noticed that most of the values (in $\mathrm{mm}$ ), for the determined dimensions, increase with age, except for certain age groups, for which they are similar or even decrease. The small sample size, when divided to age groups and the increased age intervals (1.5 years) is a possible explanation. We had insufficient data in small age groups (3.5-5 and 5-6.5) to determine En-/En and N-middle of the left pupil, due to the difficult cooperation of small children. The comparison to other anthropometric studies was difficult, as the samples have a different structure of age intervals and the used values were the mean values, which were determined for the whole sample. It suggests the need for international and interregional studies, on the same age subdivision of the sample (six months-one year), using a standardized study protocol and the same digital instruments for measurement.

Considering that, today, some powerful tools for anthropometric tridimensional analysis are available free of charge or at a limited cost, the potential impact of the method that was proposed in this study is limited. Several smartphones are able to capture threedimensional facial images, which can be analysed using freeware software, such as Viewbox (Viewbox 4, dHAL software, Kifissia, Greece), for geometric morphometric analysis [29].

\subsection{Future Perspectives}

There is a high necessity for anthropometric studies on larger samples of healthy subjects of different ages: new-borns, children in 4 to 10-year age interval, children in 10 to 15-16-year- age interval (around the peripubertal growth spurt), and young adults. The samples should be divided in six months or one year age subgroups, with a higher number of individuals for each subgroup and a balanced gender distribution. The reference values can be obtained from transversal growth studies on large samples or from longitudinal or mixed longitudinal studies.

The future growth studies should include the children and their parents in order to determine whether there are correlations between dimensions and proportions of family members; this could provide new reference values for children and adults at the same time. Three main measurements (Zy-Zy, Go-Go, and N-Gn) and two main facial indices (Facial Index and Mandibular width-face height Index) should be included in order to have an accessible assessment method, giving the possibility to analyse large samples, including transversal and longitudinal data.

Correlations between the recorded values using direct anthropometric measurements, parameters obtained by using standardized and calibrated digital photography analysed with special software, and measurements taken from 3D surfaces generated with stereophotogammetry are necessary to improve the workflow and applicability in different medical fields.

The development of interregional "Centers for dentofacial growth and development studies" also represents a need for the future perspectives.

\section{Conclusions}

In our study on healthy children and adolescents, the main anthropometric facial parameters (N-Gn, Zy-Zy, and Go-Go) increased with age. The relationship between them (as expressed by three facial indices) suffered the following changes: facial index increased with age demonstrating an increased change of the dimension $\mathrm{N}-\mathrm{G}$, when comparing to Zy-Zy; mandible-facial width index decreased demonstrating an accentuated growth of Zy-Zy comparing to Go-Go; mandibular width -face height index decreased with age, and it is related to higher dimensional change of parameter $\mathrm{N}-\mathrm{G}$, when compared to 
Go-Go. The N-Gn parameter has the highest rate of change, followed by Zy-Zy, and then by Go-Go. Some facial parameters suffer the small dimensional changes during growth and development (En-En, Al-Al, N-middle of Pupilla and Sn-Sto). The Nose Length (N-Sn), the Upper and middle figure height (Tri-Sn), and the sagittal parameters (Tr-Gn, Tr-Sn, and $\operatorname{Tr}-\mathrm{N}$ ) change significantly with age. When performing a gender related comparison, we noticed that the vertical and transversal linear parameters and some facial indices are increased in boys, depending on the age group. The correlations between facial and general growth parameters are significant in both girls and boys, except for one dimension (Sn-Sto).

We conclude that the simplified anthropometric technique represents an accessible method to every clinician, bringing important information that is related to dentofacial growth, diagnosis, and treatment planning in dentistry.

Author Contributions: Conceptualization, E.O., M.P. and K.M.; methodology, E.O., M.P. and K.M.; formal analysis, E.O., M.P., A.T. and K.M.; investigation, E.O., M.P., M.L. and K.M.; data curation, E.O., M.P., M.L., A.T. and K.M.; writing, original draft preparation-E.O., M.M., M.P., M.L., A.I. and K.M.; writing-review and editing, E.O., M.M., M.P., M.L., A.I. and K.M.; supervision, C.T. All authors have read and agreed to the published version of the manuscript.

Funding: The present study was supported by the National University Research Council of Romania (CNCSIS), BD project (Bursa Doctorala) nr. 268/2008.

Institutional Review Board Statement: The study was conducted according to the guidelines of the Declaration of Helsinki, and approved by the Ethics Committee of Victor Babes University of Medicine and Pharmacy of Timisoara, Romania (No. 04/15.09.2008).

Informed Consent Statement: Informed consent was obtained from all subjects involved in the study.

Data Availability Statement: The data presented in this study are available on request from the corresponding author.

Acknowledgments: This article was written in Memoriam of Habil. Alexandru Simion Ogodescu (1975-2020), because he dedicated much time in his short life to research, to teaching and practicing in Paedodontis\&Orthodontics field, for leading the first separate Department of Pedodontics from Timisoara (2014-2020), for his innovative ideas, for his strong belief in the importance of growth studies for our field and last, but not least, for helping us during the whole research process. The authors kindly acknowledge the parents and children who agreed to participate voluntarily to our growth studies, for their patience, thrust and for understanding the importance of updating reference values, which they made achievable.

Conflicts of Interest: The authors declare no conflict of interest.

\section{References}

1. Proffit, W.R.; Fields, H.W.; Sarver, D.M. Contemporary Orthodontics, 4th ed.; Mosby Elsevier: St. Louis, MO, USA, 2007; pp. 26-71, 167-233.

2. Enlow, D.H.; Hans, M.G. Essentials of Facial Growth; W.B. Saunders Company: Philadelphia, PA, USA, 1996; pp. 1, 5, 15, 122, $123,133-145$.

3. Bishara, S.E. Textbook of Orthodontics; W.B. Saunders Company: Philadelphia, PA, USA, 2001; pp. 31-80, 134-142.

4. Graber, T.M. Orthodontics: Principles and Practice, 3rd ed.; W.B. Saunders Company: Philadelphia, PA, USA, 1972.

5. Honn, M.; Goz, G. Reference Values for Craniofacial Structures in Children 4 to 6 Years Old: Review of the Literature. J. Orofac. Orthop. 2007, 68, 170-182. [CrossRef] [PubMed]

6. Yasas, S.N.; Jayaratne, R.; Zwahlen, A. Application of Digital Anthropometry for Craniofacial Assessment. Craniomaxillofac. Trauma Reconstr. 2014, 7, 101-107. [CrossRef]

7. Salter, E.M.; Kolar, J.C. Anthropometry. In Encyclopedia of Measurement and Statistics; Salkind, N.J., Ed.; Sage Publications: Thousand Oaks, CA, USA, 2007; Volume 1, pp. 35-38.

8. Kenneth, L.J. Smith's Recognizable Patterns of Human Malformation, 6th ed.; Elsevier Health Sciences: Philadelphia, PA, USA, 2005.

9. Bishara, S.E.; Jorgensen, G.J.; Jakobsen, J.R. Changes in facial dimensions assessed from lateral and frontal photographs. Part I-Methodology. Am. J. Orthod. Dentofac. Orthop. 1995, 108, 389-393. [CrossRef]

10. Jodeh, D.S.; Rottgers, S.A. High-Fidelity Anthropometric Facial Measurements can be obtained from a Single Stereophotograph from the Vectra H1 3-Dimensional Camera. Cleft Palate Craniofac. J. 2019, 56, 1164-1170. [CrossRef]

11. Honn, M.; Goz, G. The ideal of Facial Beauty: A Review. J. Orofac. Orthop. 2007, 68, 6-16. [CrossRef] [PubMed] 
12. Liu, H.; Li, Z.; Zheng, L. Rapid preliminary helmet shell design based on three-dimensional anthropometric head data. J. Eng. Des. 2008, 19, 45-54. [CrossRef]

13. Rushil, R.D.; Calabrese, C.E.; Burashed, H.M.; Doyle, M.; Vernacchio, L.; Resnick, C.M. Craniofacial Anthropometry: Normative Data for Caucasian Infants. J. Craniofac. Surg. 2019, 30, e539-e542. [CrossRef]

14. Kelly, K.M.; Littlefield, T.R.; Pomatto, J.K.; Ripley, C.E.; Beals, S.P.; Joganic, E.F. Importance of early recognition and treatment of deformational plagiocephaly with orthotic cranioplasty. Cleft Palate Craniofac. J. 1999, 36, 127-130. [CrossRef]

15. Asha, K.R.; Lakshmiprabha, S.; Nanjaiah, C.M.; Prashanth, S.N. Craniofacial Anthropometric Analysis in Down Syndrome. Indian J. Pediatr. 2011, 78, 1091-1095. [CrossRef]

16. Wilbrand, J.F.; Wilbrand, M.; Pons-Kuehnemann, J.; Blecher, J.C.; Christophis, P.; Howaldt, H.P.; Schaaf, H. Value and reliability of anthropometric measurements of cranial deformity in early childhood. J. Craniomaxillofac. Surg. 2011, 39, 24-29. [CrossRef]

17. De Faria, M.E.J.; Carvalho, L.R.; Rossetto, S.M.; Amaral, T.S.; Berger, K.; Arnhold, I.J.; Mendonca, B.B. Analysis of craniofacial and extremity growth in patients with growth hormone deficiency during growth hormone therapy. Horm. Res. 2009, 71, 173-177. [CrossRef]

18. Farkas, L.G.; Katic, M.J.; Forrest, C.R.; Alt, K.W.; Bagic, I.; Baltadjiev, G.; Cunha, E.; Cvicelová, M.; Davies, S.; Erasmus, I.; et al. International anthropometric study of facial morphology in various ethnic groups/races. J. Craniofac. Surg 2005, 16, 615-646. [CrossRef] [PubMed]

19. Farkas, L.G.; Katic, M.J.; Forrest, C.R. Comparison of craniofacial measurements of young adult African-American and North American white males and females. Ann. Plast. Surg. 2007, 59, 692-698. [CrossRef]

20. Arboledo, C.; Buschang, P.H.; Camacho, J.A.; Botero, P.; Roldan, S. A mixed longitudinal anthropometric study of craniofacial growth of Colombian mestizos 6-17 years of age. Eur. J. Orthod. 2011, 33, 441-449. [CrossRef] [PubMed]

21. Milicescu, V.; Milicescu, I.D. Creşterea şi Dezvoltarea Generală şi Cranio-Facială la Copii în Perioda Dentiţiei Mixte; Editura Viata Medicala Romaneasca: Bucureşti, Romania, 2001; pp. 25-27, 29-42, 46-111.

22. Firu, P. Introducere la Studiul Anomaliilor Dento-Maxilare; Ed Didactică şi Pedagogică: Bucureşti, Romania, 1983; pp. 215-227, 649-689.

23. Boboc, G. Aparatul Dento-Maxilar, Formare şi Dezvoltare, 2nd ed.; Medicală: Bucureşti, Romania, 2009; pp. 117-279.

24. Noyan, A.; Larson, B.E.; Leon-Salazar, V.; Beiraghi, S. Accuracy and precision of a 3D anthropometric facial analysis with and without landmark labelling before image acquisition. Angle Orthod. 2011, 81, 245-252. [CrossRef]

25. Baik, H.-S.; Jeon, J.-M.; Lee, H.-J. Facial soft-tissue analysis of Korean adults with normal occlusion using a 3-dimensionallaser scanner. Am. J. Orthod. Dentofac. Orthop. 2007, 131, 759-766. [CrossRef]

26. Wong, J.Y.; Oh, A.K.; Ohta, E.; Hunt, A.T.; Rogers, G.F.; Mulliken, J.B.; Deutsch, C.K. Validity and Reliability of Craniofacial Anthropometric Measurement of 3D Digital Photogrammetric Images. Cleft Palate Craniofac. J. 2008, 45, 232-239. [CrossRef]

27. Heike, C.L.; Cunningham, M.L.; Hing, A.V.; Stuhaug, E.; Starr, J.R. Picture perfect? Reliability of craniofacial anthropometry using three-dimensional digital stereophotogrammetry. Plast. Reconstr. Surg. 2009, 124, 1261-1272. [CrossRef] [PubMed]

28. Sforza, C.; Laino, A.; D’Alessio, R.; Grandi, G.; Catti, F.; Ferrario, V.F. Three-dimensional facial morphometry of attractive adolescent boys and girls. Prog. Orthod. 2007, 8, 268-281.

29. Kanavakis, G.; Halazonetis, D.; Katsaros, C.; Gkantidis, N. Facial shape affects self-perceived facial attractiveness. PLoS ONE 2021, 16, e0245557. [CrossRef]

30. Othman, S.A.; Majawit, L.P.; Wan Hassan, W.N.; Wey, M.C.; Mohd Razi, R. Anthropometric Study of Three-Dimensional Facial Morphology in Malay Adults. PLoS ONE 2016, 11, e0164180. [CrossRef]

31. Fourie, Z.; Damstra, J.; Gerrits, P.O.; Ren, Y. Evaluation of anthropometric accuracy and reliability using different threedimensional scanning systems. Forensic Sci. Int. 2011, 207, 127-134. [CrossRef] [PubMed]

32. Kukharev, G.A.; Kaziyeva, N. Digital Facial Anthropometry: Application and Implementation. Pattern Recognit. Image Anal. 2020, 30, 496-511. [CrossRef]

33. Menéndez López-Mateos, M.L.; Carreño-Carreño, J.; Palma, J.C.; Alarcón, J.A.; Menéndez López-Mateos, C.; Menéndez-Núñez, M. Three-dimensional photographic analysis of the face in European adults from southern Spain with normal occlusion: Reference anthropometric measurements. BMC Oral Health 2019, 19, 196. [CrossRef]

34. Flores, M.R.P.; Machado, C.E.P.; Gallidabino, M.D.; de Arruda, G.H.M.; da Silva, R.H.A.; de Vidal, F.B.; Melani, R.F.H. Comparative Assessment of a Novel Photo-Anthropometric Landmark-Positioning Approach for the Analysis of Facial Structures on TwoDimensional Images. J. Forensic Sci. 2019, 64, 828-838. [CrossRef]

35. Krimmel, M.; Breidt, M.; Bacher, M.; Müller-Hagedorn, S.; Dietz, K.; Bülthoff, H.; Reinert, S.; Kluba, S. Three-Dimensional Normal Facial Growth from Birth to the Age of 7 Years. Plast. Reconstr. Surg. 2015, 136, 490e-501e. [CrossRef] [PubMed]

36. Machado, C.E.P.; Flores, M.R.P.; Lima, L.N.C.; Tinoco, R.L.R.; Franco, A.; Bezerra, A.C.B.; Evison, M.P.; Guimarães, M.A. A new approach for the analysis of facial growth and age estimation: Iris ratio. PLoS ONE 2017, 12, e0180330. [CrossRef] [PubMed]

37. Ionescu, E.; Milicescu, I.D.; Popescu, M.; Popoviciu, O.; Milicescu, V. Ortodonţie şi Ortopedie Dento-Facială. In Ghid Clinic şi Terapeutic (Pentru Studenţi şi Rezidenţi); Ed. Cerma: Bucureşti, Romania, 2001; pp. 33-38, 75-137.

38. Matthew, J.K.; Zachary, D.R.; Carrie, L.H.; Michael, L.C.; Jacqueline, T.H.; Chung, H.K.; Nidey, N.L.; Moreno, L.M.; Wehby, G.L.; Marazita, M.L.; et al. Using the 3D Facial Norms Database to investigate craniofacial sexual dimorphism in healthy children, adolescents, and adults. Biol. Sex Differ. 2016, 7, 23. [CrossRef]

39. Farkas, L.G.; Hreczko, T.M.; Katic, M.J.; Forrest, C.R. Proportion indices in the craniofacial regions of 284 healthy North American white children between 1 and 5 years of age. J. Craniofac. Surg. 2003, 14, 13-28. [CrossRef] 
40. Valean, C.; Tatar, S.; Nanulescu, M.; Leucuta, A.; Ichim, G. Prevalence of obesity and overweight among school children in Cluj-Napoca. Acta Endocrinol. 2009, 5, 213-219. [CrossRef]

41. Moldovanu, F.; Nanu, M.; Ardeleanu, I.; Stativa, E.; Nanu IBacalearos, C. Overweight and obesity in Romanian children, in European context. Arch. Dis. Child. 2017, 102 (Suppl. 2), A1-A192. [CrossRef]

42. Chirita-Emandi, A.; Barbu, C.G.; Cinteza, E.E.; Chesaru, B.I.; Gafencu, M.; Mocanu, V.; Pascanu, I.M.; Tatar, S.A.; Balgradean, M.; Dobre, M.; et al. Overweight and Underweight Prevalence Trends in Children from Romania-Pooled Analysis of Cross-Sectional Studies between 2006 and 2015. Obes. Facts 2016, 9, 206-220. [CrossRef]

43. Negeow, W.C.; Aljunid, S.T. Craniofacial anthropometric norms of Malays. Singap. Med. J. 2009, 50, 525-528.

44. Agnihotri, A.K.; Kachhwaha, S.; Googoolye, K.; Allock, A. Estimation of stature from cephalo-facial dimensions by regression analysis in Indo-Mauritian population. J. Forensic Leg. Med. 2011, 18, 167-172. [CrossRef]

45. Krishan, K.; Kumar, R. Determination of stature from cephalo-facial dimensions in a North Indian population. Leg. Med. 2007, 9, 128-133. [CrossRef] [PubMed] 